\title{
Myocardial blood flow quantification by Rb-82 cardiac PET/CT: Methodological issues on reproducibility study
}

I was interested to read the paper by Dunet $\mathrm{V}$ and colleagues published in the Jun 2016 issue of $\mathrm{J} \mathrm{Nucl}$ Cardiol. ${ }^{1}$ They aimed to assess reproducibility of two software packages (PMOD and FlowQuant (Lortie model)) for quantifying myocardial blood flow (MBF) and myocardial flow reserve (MFR) using dynamic $\mathrm{Rb}$ 82 PET at rest and during adenosine stress. ${ }^{1}$

They used Pearson's correlation $\rho$ for measuring precision and reported an excellent precision $\rho$ for MBF $(\rho=0.97)$ and good precision for MFR $(\rho=0.83)$. However, PMOD provided slightly higher values than FlowQuant at higher MBF and MFR values $(P<.0001) .{ }^{1}$

Reproducibility (precision, repeatability, reliability, or interchangeability) is being assessed by different statistical tests including Pearson's correlation $\rho$ which is one of the common mistakes in Reproducibility analysis. ${ }^{2-6}$ Pearson's correlation $\rho$ only assesses the linearity between two continuous variables. Any shift in the location and/or scale of the regression line which leads to nonreproducibility cannot be detected by this correlation coefficient. ${ }^{2}$ Therefore, for quantitative variables, Intra Class Correlation Coefficient single measure is the best statistical test to evaluate reproducibility. ${ }^{2-6}$ It is good to know that in reliability analysis individual approach should be considered instead of global average which Pearson's correlation $\rho$ cannot do that. Moreover, statistically significant should not be considered in reproducibility analysis. ${ }^{3-6}$ As a take home message, for reliability analysis, appropriate tests should be applied, otherwise, misdiagnosis and mismanagement of the patients cannot be avoided.
Disclosure The authors have indicated that they have no financial conflict of interest.

$$
\begin{array}{r}
\text { Siamak Sabour, MD, MSc, DSc, PhD, } \\
\text { Postdoc }{ }^{a, b}, \\
\text { Safety Promotion and Injury Prevention } \\
\text { Research Center, Shahid Beheshti } \\
\text { University of Medical Sciences, Tehran, } \\
\text { Islamic Republic of Iran; } \\
\text { Department of Clinical Epidemiology, } \\
\text { School of Health, Shahid Beheshti } \\
\text { University of Medical Sciences, Tehran, } \\
\text { Islamic Republic of Iran } \\
\text { s.sabour@sbmu.ac.ir. }
\end{array}
$$

\section{References}

1. Dunet V, Klein R, Allenbach G, Renaud J, deKemp RA, Prior JO. Myocardial blood flow quantification by Rb-82 cardiac PET/CT: A detailed reproducibility study between two semi-automatic analysis programs. J Nucl Cardiol. 2016;23:499-510. doi:10.1007/s12350015-0151-2.

2. Lawrence I, Lin K. A concordance correlation coefficient to evaluate reproducibility. BIOMETRICS. 1989;45:255-68.

3. Rothman KJ, Greenland S, Lash TL. Modern Epidemiology. Baltimore: Lippincott Williams \& Wilkins; 2010.

4. Sabour S. Spinal instability neoplastic scale: Methodologic issues to avoid misinterpretation. AJR Am J Roentgenol. 2015;204:W493. doi:10.2214/AJR.14.13870

5. Sabour S. Reliability of automatic vibratory equipment for ultrasonic strain measurement of the median nerve: Common mistake. Ultrasound Med Biol. 2015;41:1119-20. doi:10.1016/j.ultrasmedbio. 2014.10.017

6. Sabour S. Methodologic concerns in reliability of noncalcified coronary artery plaque burden quantification. AJR Am J Roentgenol. 2014;203:W343. doi:10.2214/AJR.14.12649.

doi:10.1007/s12350-016-0577-1

Funding None 